\title{
ON PERMUTATION REPRESENTATIONS
}

\author{
BY \\ Alex Heller \\ In Memory of Irving Reiner
}

By a permutation representation $(G, X)$ we mean a group $G$ together with a left $G$-set $X$. The orbit set $G \backslash X$ has as its elements the orbits $[x]=G x$ for $x \in X$. The question which we shall address is to what extent the permutation representation can be recovered from information about orbit sets alone. Its motivation comes from homotopy theory and we shall indicate below how our remarks apply there.

Evidently from $G \backslash X$ alone we cannot reconstruct $G$ and $X$. But for any set $U$ the $U$-th power of $X$, i.e., the set $X^{U}$ of functions $x: U \rightarrow X$, is again a $G$-set, with $(g x) i=g(x i)$ for $g \in G, i \in U$. Furthermore if $F: U \rightarrow V$ then $X^{f}: X^{V} \rightarrow X^{U}$, the composition with $f$, is a $G$-equivariant map. Thus

$$
U \mapsto G \backslash X^{U}, f \mapsto G \backslash X^{f}
$$

defines a functor $\operatorname{Orb}(G, X):$ Sets ${ }^{\text {op }} \rightarrow$ Sets, the orbit-functor of $(G, X)$. We shall see that from this functor we can indeed reconstruct, up to a suitable equivalence, the permutation representation.

\section{Orbital functors}

We shall adopt the following conventions. A natural number $n$ is the set $\{0,1, \ldots, n-1\}$ of its predecessors, so that $0=\varnothing$. If $f: m \rightarrow m^{\prime}$ and $g$ : $n \rightarrow n^{\prime}$ are maps of natural numbers then $f+g: m+n \rightarrow m^{\prime}+n^{\prime}$ is the ordinal sum in the obvious sense. For any set $W, \underline{W}: 0 \rightarrow W$ and $\bar{W}: W \rightarrow 1$ are the unique maps; we shall also on occasion write $W$ for the identity map. For sets $U, V, \Delta: U \rightarrow U^{V}$ denotes the generalized diagonal map. If $F$ : Sets $^{\text {op }} \rightarrow$ Sets then the natural transformation dg: $F(U \times V) \rightarrow(F U)^{V}$ is defined by $\operatorname{pr}_{j} \mathrm{dg}=F(U \times j)$ where $j: 1 \rightarrow V$ is an element of $V$.

Received September 22, 1987.

(C) 1988 by the Board of Trustees of the University of Illinois Manufactured in the United States of America 
If $Y: 1 \rightarrow F U$ is an element of $F U$ we define the functor

$$
F_{Y}: \text { Sets }^{\text {op }} \times \text { Sets }^{\text {op }} \rightarrow \text { Sets, }
$$

which we shall (a bit fancifully) call the functor tangent to $F$ at $Y$, by taking for $F_{Y}(V, W)$ the subset of $F(U \times V \sqcup W)$ such that

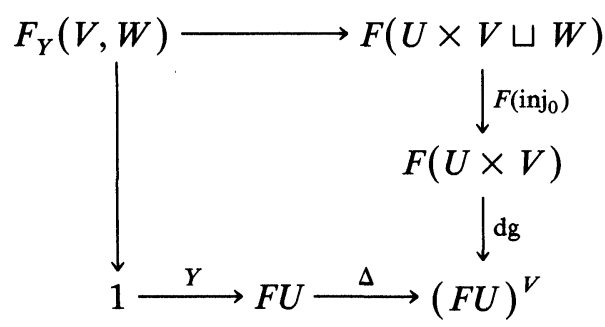

is a pullback.

Note that $F_{Y}(0, W)=F W$, while $F_{Y}(1,0)=\{Y\} \approx 1$.

An element $Y \in F U$ is generic if $F_{Y}$ satisfies the following conditions:

(G1) For $n=1,2,3$ the maps

$$
\begin{aligned}
\alpha_{n}(W) & =\left\langle F_{Y}(2+\underline{n-1}, \underline{W}), F_{Y}(\underline{1}+n, W)\right\rangle: \\
F_{Y}(n+1, W) & \rightarrow F_{Y}(2,0) \times F_{Y}(n, W)
\end{aligned}
$$

are bijective.

(G2) The diagrams

$$
F_{Y}(2, W) \underset{F_{Y}(1+\underline{1}, W)}{\stackrel{F_{Y}(\underline{1}+1, W)}{\longrightarrow}} F_{Y}(1, W) \stackrel{F_{Y}(\underline{1}, W)}{\longrightarrow} F_{Y}(0, W)
$$

are coequalizers.

(G3) The maps dg: $F_{Y}(1, W) \rightarrow F_{Y}(1,1)^{W}$ are bijective.

A functor $F$ is orbital if it has a generic element. If $Y \in F U$ is generic then

$$
\begin{aligned}
\mu & =F_{Y}(1+\underline{1}+1,0) \alpha_{2}(0)^{-1}: F_{Y}(2,0) \times F_{Y}(2,0) \rightarrow F_{Y}(2,0) \\
\iota & =F_{Y}(\operatorname{tr}, 0): F_{Y}(2,0) \rightarrow F_{Y}(2,0), \text { where tr is the transposition, } \\
\varepsilon & =F_{Y}(\overline{2}, 0): F_{Y}(1,0) \approx F_{Y}(1,1)^{0} \approx 1 \rightarrow F_{Y}(2,0)
\end{aligned}
$$

are, respectively, binary, unary and 0 -ary operations in the set $F_{Y}(2,0)$ and

$$
\xi_{W}=F_{Y}(1+\underline{1}, W) \alpha_{1}(W)^{-1}: F_{Y}(2,0) \times F_{Y}(1, W) \rightarrow F_{Y}(1, W)
$$

is a natural transformation. 
Proposition 1.1. (i) The operations $\mu, \iota, \varepsilon$ give to $F_{Y}(2,0)$ the structure of a group.

(ii) $\xi_{W}$ makes $F_{Y}(1, W)$ a left $F_{Y}(2,0)$-set and, if $f: W \rightarrow W^{\prime}, F_{Y}(1, f)$ an $F_{Y}(2,0)$-equivariant map.

(iii) Thus, as left $F_{Y}(2,0)$ sets,

$$
\begin{aligned}
F_{Y}(1, W) & \approx F_{Y}(1,1)^{W} \text { and } \\
F W & \approx F_{Y}(2,0) \backslash F_{Y}(1, W) \approx \operatorname{Orb}\left(F_{Y}(2,0), F_{Y}(1,1)\right) W .
\end{aligned}
$$

This is proved by straightforward if tedious computation which need not be carried out in detail here. Indeed that part of (i) which asserts that $\mu, \varepsilon$ give a monoid structure on $F_{Y}(2,0)$ will be recognized as a standard exercise in category theory once it is observed that $[n] \rightarrow F_{Y}(n+1,0)$ is a simplicial set. The rest of the argument is in the same spirit.

We shall write $\operatorname{Pr}_{Y} F$ for the permutation representation $\left(F_{Y}(2,0), F_{Y}(1,1)\right)$.

\section{Orbit functors}

If $(G, X)$ is a permutation representation and $x \in X$ we write $J_{x}$ for the inertial subgroup $\{g \mid g x=x\}$ of $G$ at $x$. Thus the orbit $[x]$ is free if and only if $J_{x}$ is the trivial subgroup and $(G, X)$ is a faithful representation if and only if $N=\bigcap_{x \in X} J_{x}$ is trivial. Since $N$ is a normal subgroup operating trivially on $X$ the quotient $\tilde{G}=G / N$ still operates on $X$, so that $(\tilde{G}, X)$ is a faithful permutation representation. Evidently $\operatorname{Orb}(\tilde{G}, X)=\operatorname{Orb}(G, X)$.

It will be no surprise that $\operatorname{Orb}(G, X)$ is an orbital functor. The following proposition also provides a simple criterion for genericity.

Proposition 2.1. Let $(G, X)$ be a faithful permutation representation and set $F=\operatorname{Orb}(G, X)$. If $Y \in F U$ the following are equivalent:

(i) $Y$ is a free orbit;

(ii) $Y$ is generic;

(iii) $\mathrm{dg}: F_{Y}(1,2) \rightarrow F_{Y}(1,1)^{2}$ is bijective.

A moment's thought shows that the functor tangent to $F$ at $Y$ is given by

$$
F_{Y}(V, W)=G \backslash\left(Y^{V} \times X^{W}\right) .
$$

Suppose that $Y$ is free and choose some $y \in Y$. We may then evaluate $F_{Y}(V \sqcup 1, W)$ in the following way. For any $(w, x) \in Y^{V \sqcup 1} \times X^{W}$ there is a unique $g \in G$ such that $g w_{0}=y$. Thus $(w, x) \mapsto(g(w \mid V), g x)$ defines a map which factors uniquely through a natural bijection $\varphi_{y, \mathrm{v}, \mathrm{w}}: F_{Y}(V \sqcup 1, W) \rightarrow$ $Y^{V} \times X^{W}$. The conditions G1-G3 follow easily, so that (i) implies (ii). 
That (ii) implies (iii) is immediate, since the latter is a special case of G3. Now suppose that $Y=[y]$ is not free. Then there are, since $(G, X)$ is faithful, an $x \in X$ and a $g \in G$ such that $g y=y, g x \neq x$. Thus $[y, x, g x] \neq[y, x, x]$ while $[y, x]=[y, g x]$. In other words,

$$
\operatorname{dg}[y, x, g x]=\operatorname{dg}[y, x, x]=([y, x],[y, x]),
$$

violating (iii).

Corollary 2.2. If $(G, X)$ is a permutation representation then $\operatorname{Orb}(G, X)$ is orbital.

Without loss of generality, we may suppose $(G, X)$ faithful. Then it is only necessary to see that some $X^{U}$ contains a free orbit. But, for any $x \in X^{U}, J_{x}=$ $\cap_{i \in U} J_{x i}$. Thus $\left[\mathrm{id}_{X}\right] \in F X$ is free.

Let us suppose that $Y=[y] \in F U$ is a free orbit where, once again, $F=\operatorname{Orb}(G, X)$. Then $\varphi_{y, 0,1}: F_{Y}(1,1) \approx X$ and $\varphi_{y, 1,0}: F_{Y}(2,0) \approx Y$. Let us abbreviate $\left(\varphi_{y, 0,1}\right)^{-1}$ by $\psi_{y}$ and write $\vartheta_{y}$ for the composition of $\left(\varphi_{y, 1,0}\right)^{-1}$ with the bijection $g \mapsto g y$ of $G$ to $Y$. Then $\vartheta_{y}$ is an isomorphism of groups and $\psi_{y}$ is a $\vartheta_{y}$-equivariant bijection. For the multiplication in $F_{Y}(2,0)$ is just

$$
([y, g y],[y, h y])=([y, g y],[g y, g h y]) \mapsto[y, g y, g h y] \mapsto[y, g h y],
$$

while the operation of $F_{Y}(2,0)$ on $F_{Y}(1,1)$ is

$$
([y, g y],[y, x])=([y, g y],[g y, g x]) \mapsto[y, g y, g x] \mapsto[y, g x] .
$$

We have, accordingly, proved the following statement.

Proposition 2.3. If $(G, X)$ is a faithful permutation representation and $Y=[y]$ is a free orbit in some $X^{U}$ then $\left(\vartheta_{y}, \psi_{y}\right):(G, X) \mapsto \operatorname{Pr}_{Y} \operatorname{Orb}(G, X)$ is an isomorphism of permutation representations.

COROllary 2.4. If $(G, X)$ is a (not necessarily faithful) permutation representation then $\operatorname{Pr}_{Y} \operatorname{Orb}(G, X) \approx(\tilde{G}, X)$. If $F$ is an orbital functor then $\operatorname{Pr}_{Y} F$ is a faithful permutation representation.

\section{Functoriality}

We have seen how a faithful permutation representation $(G, X)$ can be recovered, up to isomorphism, from its orbit functor $\operatorname{Orb}(G, X)$. What remains is to explicate the meaning of "up to isomorphism."

Let us denote by PR the category (actually, the groupoid) whose objects are faithful permutation representations and whose morphisms are isomorphisms $(\vartheta, \psi):(G, X) \rightarrow\left(G^{\prime}, X^{\prime}\right)$. If $g \in G^{\prime}$ and $C_{g}$ denotes conjugation by $g$ then 
$\left(C_{g} \vartheta, g \psi\right)$ is again an isomorphism. The relation $(\vartheta, \psi) \sim\left(C_{g} \vartheta, g \psi\right)$ is a congruence in PR; we denote the quotient category by $\overline{\mathrm{PR}}$.

The orbital functors Sets ${ }^{\mathrm{op}} \rightarrow$ Sets and the natural isomorphisms between them also constitute a category: since (G2), (G3) assert that any orbital functor is a quotient of a representable functor the natural isomorphisms $F \rightarrow F^{\prime}$ form a set. Let us denote this category by ORB.

If $(\vartheta, \psi):(G, X) \rightarrow\left(G^{\prime}, X^{\prime}\right)$ then $[x] \rightarrow[\psi x]$ gives an isomorphism

$$
(\vartheta, \psi)_{*}: \operatorname{Orb}(G, X) \rightarrow \operatorname{Orb}\left(G^{\prime}, X^{\prime}\right)
$$

If $(\boldsymbol{\vartheta}, \psi) \sim\left(\boldsymbol{\vartheta}^{\prime}, \psi^{\prime}\right)$ then $(\boldsymbol{\vartheta}, \psi)_{*}=\left(\boldsymbol{\vartheta}^{\prime}, \psi^{\prime}\right)_{*}$. Thus Orb defines a functor $\overline{\mathrm{PR}} \rightarrow \mathrm{ORB}$. The results of $\S 1,2$ are then summed up and amplified by the following theorem.

THEOREM 3.1. Orb: $\overline{\mathrm{PR}} \rightarrow \mathrm{ORB}$ is an equivalence of categories.

Suppose that $F$ : Sets ${ }^{\mathrm{op}} \rightarrow$ Sets, $f: U^{\prime} \rightarrow U, Y \in F U$ and $Y^{\prime}=(F f) Y$. Then, for any $V, W, F(f \times V \sqcup W): F(U \times V \sqcup W) \rightarrow F\left(U^{\prime} \times V \sqcup W\right)$ takes $F_{Y}(V, W)$ into $F_{Y^{\prime}}(V, W)$ and its restriction $f^{*}$ is a natural transformation $F_{Y} \rightarrow F_{Y^{\prime}}$. If $F$ is an orbital functor then by (1.1) it is isomorphic to an orbit functor and from (2.1) we reach easily the following conclusion.

LEMMA 3.2. If $F$ is an orbital functor, $f: U^{\prime} \rightarrow U, Y \in F U$ and $Y^{\prime}=(F f) Y$ is generic then $Y$ is also generic and $f^{*}: F_{Y} \rightarrow F_{Y^{\prime}}$ is an isomorphism. In particular $f^{*}$ gives an isomorphism $\left(f_{2,0}^{*}, f_{1,1}^{*}\right): \operatorname{Pr}_{Y} F \rightarrow \operatorname{Pr}_{Y^{\prime}} F$.

Now suppose, in the situation just described, that there is another map $f^{\prime}$ : $U^{\prime} \rightarrow U$ such that $\left(F f^{\prime}\right) Y=Y^{\prime}$. Then $\left(f, f^{\prime}\right): U^{\prime} \sqcup U^{\prime} \rightarrow U$ determines a map

$$
1 \approx F_{Y}(1,0) \rightarrow F_{Y^{\prime}}(2,0)
$$

i.e., an element $g \in F_{Y^{\prime}}(2,0)$. An easy calculation shows that $f_{1,1}^{*}=g f_{1,1}^{\prime *}$ and $f_{2,0}^{*}=C_{g} f_{2,0}^{\prime *}$, so that

$$
\left(f_{2,0}^{*}, f_{1,1}^{*}\right) \sim\left(f_{2,0}^{\prime *}, f_{1,1}^{\prime *}\right)
$$

Furthermore $F\left(U \sqcup U^{\prime}\right) \rightarrow F U \times F U^{\prime}$ is patently surjective. Thus given generic $Y \in F U, Y^{\prime} \in F U^{\prime}$ there is a $Y^{\prime \prime} \in F U^{\prime \prime}$ mapping to both and, in view of our previous observation, a unique morphism $\operatorname{Pr}_{Y} F \rightarrow \operatorname{Pr}_{Y^{\prime}} F$, determined by suitable $\left(f_{2,0}{ }^{*}, f_{1,1}{ }^{*}\right)$ but independent of the choices of the maps $f$. We have accordingly proved the following assertion. 
LEMMA 3.3. If $F$ is an orbital functor then the permutation representations $\operatorname{Pr}_{Y} F$ and the isomorphisms generated by $\left\{\left(f_{2}{ }^{*}, 0, f_{1}{ }^{*}\right)\right\}$ form an indiscrete subgroupoid $\operatorname{Pr} F \subset \overline{\mathrm{PR}}$.

Finally, if $(G, X)$ is a faithful permutation representation then for any free orbit $Y=[y]$ the isomorphism $\left(\vartheta_{y}, \psi_{y}\right):(G, X) \rightarrow \operatorname{Pr}_{Y} \operatorname{Orb}(G, X)$ of (2.3) is, in $\overline{\mathrm{PR}}$, independent of $y$, since $\left(\vartheta_{g y}, \psi_{g y}\right)=\left(C_{g} \vartheta_{y}, g \psi_{y}\right) \sim\left(\vartheta_{y}, \psi_{y}\right)$. If we denote by $(\vartheta, \psi)_{Y}$ the class of $\left(\vartheta_{y}, \psi_{y}\right)$ in PR then it is clear that, for any free orbits $Y, Y^{\prime}, \alpha(\vartheta, \psi)_{Y}=(\vartheta, \psi)_{Y^{\prime}}$, where $\alpha: \operatorname{Pr}_{Y} F \rightarrow \operatorname{Pr}_{Y^{\prime}} F$ is the unique morphism in $\operatorname{Pr} F$.

Theorem 3.1 now follows immediately.

\section{Algebraic permutation representations}

It is appropriate to consider permutation representations $(G, X)$ in which the set $X$ has additional structure, preserved by the action of $G$. In particular we shall turn our attention to the case that $X$ has an algebraic structure, such as that of a group or ring or module over some ring - this last case bringing us within the ambit of classical representation theory.

Such an algebraic structure in $X$ is provided by a family of finitary operations, an $n$-ary operation, $n=0,1,2, \ldots$, being a map $X^{n} \rightarrow X$. The action of $G$ preserves this algebraic structure when each of the operations is an equivariant map. We say that such an algebraic structure is invariant.

The problem with which we began, viz. how to recover $(G, X)$ from its orbit structure, now exhibits an ambiguity, namely, in the interpretation to be accorded to the "orbit-set" $G \backslash X$. This may, for example, be interpreted as the coequalizer in the appropriate category of algebras of all the translations in $G$. In this case, however, the methods we have used above are inappropriate. We shall accordingly not discuss it here.

Alternatively we may continue to mean by $G \backslash X$ the orbit-set in the sense we have adopted above. However the algebraic structure in $X$ does not in general pass to the orbit-sets $G \backslash X^{U}$, and we must thus look elsewhere for the relevant information.

By an $n$-ary operation in a functor $F:$ Sets ${ }^{\text {op }} \rightarrow$ Sets we mean a natural transformation

$$
\omega_{U}: F(U \sqcup n) \rightarrow F(U \sqcup 1) .
$$

If $F$ is orbital with generic $Y \in F U$ then $\omega_{U \times V}$ restricts to a natural transformation $F_{Y}(V, n) \rightarrow F_{Y}(V, 1)$ and thus yields an $F_{Y}(2,0)$-equivariant map

$$
\hat{\omega}: F_{Y}(1,1)^{n} \approx F_{Y}(1, n) \rightarrow F_{Y}(1,1),
$$

which is to say an equivariant $n$-ary operation on $F_{Y}(1,1)$. 
Conversely if $(G, X)$ is a faithful permutation representation and is an equivariant $n$-ary operation on $X$ then commutativity in

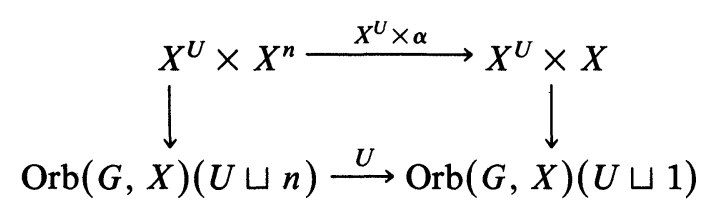

defines an $n$-ary operation in $\operatorname{Orb}(G, X)$. Straightforward calculation shows that for any free orbit $Y=[y]$ in, say, $X^{U}$, the isomorphism

$$
(G, X) \rightarrow \operatorname{Pr}_{Y} \operatorname{Orb}(G, X)
$$

of Proposition 2.3 takes $\alpha$ to $\alpha^{\vee \wedge}$.

Similarly, for an operation $\omega$ in an orbital functor $F$, the canonical isomorphism $F \rightarrow \operatorname{Orb}\left(F_{Y}(2,0), F_{Y}(1,1)\right)$ of $(1.1)$ takes $\omega$ to $\omega^{\wedge \vee}$. We have thus proved the following assertion.

Proposition 4.1. Let $(G, X)$ be a permutation representation. Then finitary equivariant operations in $X$ correspond bijectively (under ${ }^{\wedge},{ }^{\vee}$ ) to operations in $\operatorname{Orb}(G, X)$.

In effect we have learned how to recover an invariant algebraic structure on $X$ from a corresponding structure in $\operatorname{Orb}(G, X)$. It is only the lack of an adequate vocabulary for universal algebra which prevents us, at this point, from asserting the appropriate generalization of Theorem 3.1. The vocabulary is already in existence-in several versions - and there seems to be no point in reintroducing it here.

\section{Relative homotopy}

If $\mathfrak{X}$ and $\mathfrak{Y}$ are topological spaces we denote by $\Pi(\mathfrak{X}, \mathfrak{Y})$ the set of homotopy classes of maps $\mathfrak{X} \rightarrow \mathfrak{Y}$. If $j: \mathfrak{A} \rightarrow \mathfrak{X}$ is the inclusion of a cofibered subspace then $\Pi(j, \mathfrak{Y}): \Pi(\mathfrak{X}, \mathfrak{V}) \rightarrow \Pi(\mathfrak{A}, \mathfrak{Y})$ and, if $\alpha \in \Pi(\mathfrak{A}, \mathfrak{Y})$, we set $\Pi_{\alpha}(\mathfrak{X}, \mathfrak{V})=\Pi(j, \mathfrak{V})^{-1} \alpha$.

Thus free homotopy is to be contrasted with relative homotopy. Suppose a: $\mathfrak{U} \rightarrow \mathfrak{Y}$. Then maps $f_{0}, f_{1}: \mathfrak{X} \rightarrow \mathfrak{V}$ with $f_{i} j=a$ are homotopic $($ rel $\mathfrak{U})$ if there is a homotopy between them which is stationary on $\mathfrak{A}$. This is an equivalence relation and we denote the set of equivalence classes by $\Pi(\mathfrak{X}, \mathfrak{Y}$; rel $a)$. Evidently relative homotopy implies homotopy, giving a map

$$
\Pi(\mathfrak{X}, \mathfrak{V} ; \operatorname{rel} a) \rightarrow \Pi_{\alpha}(\mathfrak{X}, \mathfrak{Y})
$$

where $\alpha$ is the homotopy class of $a$. 
Relative homotopy and free homotopy are related in the following way. We regard the 1-sphere $S^{1}$ as a pointed space, giving a standard imbedding $\mathfrak{A} \rightarrow \mathfrak{A} \times S^{1}$.

Proposition 5.2. If a: $\mathfrak{A} \rightarrow \mathfrak{Y}$ then $\Pi\left(\mathfrak{A} \times S^{1}, \mathfrak{Y}\right.$; rel $\left.a\right)$ has the structure of a group. If also $\mathfrak{A}$ is imbedded as a cofibered subspace of $X$ then this group operates on $\Pi(\mathfrak{X}, \mathfrak{Y}: \mathrm{rel} a)$ and the orbit set is given by the map (5.1).

In other words, $\Pi_{\alpha}(\mathfrak{X}, \mathfrak{Y}) \approx \Pi\left(\mathfrak{A} \times S^{1}, \mathfrak{Y}:\right.$ rel $\left.a\right) \backslash \Pi(\mathfrak{X}, \mathfrak{Y} ;$ rel $a)$.

The proof is an easy exercise and need not be detailed here, except to note that the group multiplication comes from the usual map $S^{1} \rightarrow S^{1} \vee S^{1}$.

In the light of what we have done above, we may now, rather surprisingly, adduce a computation in the reverse direction, thus deriving relative homotopy, up to isomorphism, from free homotopy.

For any set $U$, regarded as a discrete space, define $U \times_{\mathfrak{A}} \mathfrak{X}$ by the pushout

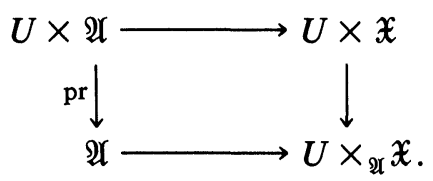

It is easy to see that $\Pi\left(U \times_{\mathfrak{Y}} \mathfrak{X}, \mathfrak{V} ; \operatorname{rel} a\right) \approx \Pi(\mathfrak{X}, \mathfrak{V} ; \operatorname{rel} a)^{U}$. Thus by (5.2),

$$
\Pi_{\alpha}\left(U \times_{\mathfrak{A}} \mathfrak{X}, \mathfrak{Y}\right) \approx \operatorname{Orb}\left(\Pi\left(\mathfrak{A} \times S^{1}, \mathfrak{Y} ; \operatorname{rel} a\right), \Pi(\mathfrak{X}, \mathfrak{Y} ; \operatorname{rel} a)\right)^{U} .
$$

In other words the orbit functor is computable in terms of free homotopy.

THEOREM 5.3. The functor $F$ defined by $F U=\Pi_{\alpha}\left(U \times_{\mathfrak{H}} \mathfrak{X}, \mathfrak{Y}\right)$ is orbital and for any generic $Y \in F U$ there is an isomorphism of permutation representations

$$
\left(\Pi\left(\mathfrak{A} \times S^{1}, \mathfrak{Y} ; \text { rel } a\right)^{\sim}, \Pi(\mathfrak{X}, \mathfrak{Y} ; \operatorname{rel} a)\right) \approx \operatorname{Pr}_{Y} F .
$$

In particular, $\Pi(\mathfrak{X}, \mathfrak{Y} ;$ rel $a) \approx F_{Y}(1,1)$.

The case in which $\mathfrak{A}=*$ consists of just one point, that of pointed homotopy, is of particular interest. A map $y: * \rightarrow \mathfrak{V}$ is just a point of $\mathfrak{Y}$ and $\Pi\left(* \times S^{1}, \mathfrak{V}\right.$; rel $\left.y\right)$ is just $\pi_{1}(\mathfrak{V}, y)$. If further $\mathfrak{V}$ is path-connected then $\Pi(*, \mathfrak{Y})$ has just one element and we may drop the subscript $\alpha$ which appears above.

If $X=S^{n}, n=1,2, \ldots$ then $\Pi(\mathfrak{X}, \mathfrak{V}$; rel $y)=\pi_{n}(\mathfrak{Y}, y)$ which is of course a group, abelian if $n>1$. The operation of $\pi_{1}(\mathfrak{V}, y)$ is the familiar one. The defining operations of $\pi_{n}(\mathfrak{V}, y)$ come from maps

$$
S^{n} \rightarrow S^{n} \vee S^{n}, \quad S^{n} \rightarrow S^{n}, \quad * \rightarrow S^{n}
$$


and the corresponding operations in the orbital functor $F U=\Pi\left(U \times{ }_{*} S^{n}, \mathfrak{V}\right)$ are thus themselves computable in free homotopy.

THEOREM 5.4. For any generic $Y \in F U$,

$$
\left(\pi_{1}(\mathfrak{Y}, y)^{\tilde{1}}, \pi_{n}(\mathfrak{Y}, y)\right) \approx \operatorname{Pr}_{Y} F
$$

as algebraic permutation representations.

City University OF NeW YoRK

NEW YORK 